\title{
Detection of invisible cracks in ceramic materials using by pre-trained deep convolutional neural network
}

\author{
Hidir Selcuk Nogay ${ }^{1} \cdot$ Tahir Cetin Akinci, ${ }^{2,3}$ (D) Musa Yilmaz ${ }^{4}$
}

Received: 23 June 2020 / Accepted: 26 October 2021 / Published online: 8 November 2021

(c) The Author(s) 2021

\begin{abstract}
Ceramic materials are an indispensable part of our lives. Today, ceramic materials are mainly used in construction and kitchenware production. The fact that some deformations cannot be seen with the naked eye in the ceramic industry leads to a loss of time in the detection of deformations in the products. Delays that may occur in the elimination of deformations and in the planning of the production process cause the products with deformation to be excessive, which adversely affects the quality. In this study, a deep learning model based on acoustic noise data and transfer learning techniques was designed to detect cracks in ceramic plates. In order to create a data set, noise curves were obtained by applying the same magnitude impact to the ceramic experiment plates by impact pendulum. For experimental application, ceramic plates with three invisible cracks and one undamaged ceramic plate were used. The deep learning model was trained and tested for crack detection in ceramic plates by the data set obtained from the noise graphs. As a result, $99.50 \%$ accuracy was achieved with the deep learning model based on acoustic noise.
\end{abstract}

Keywords Acoustic noise curves · Pulse pendulum - Transfer learning · Deep convolutional neural network · Alexnet

\section{Introduction}

Increasing the variety of quality products and reducing costs in the ceramic industry is economically prioritized and important. Different types of deformations are seen at every stage of production due to multiple reasons. The

Tahir Cetin Akinci

tahircetin.akinci@ucr.edu; akincitc@itu.edu.tr

Hidir Selcuk Nogay

nogay@kayseri.edu.tr

Musa Yilmaz

musa.yilmaz@batman.edu.tr

1 Department of Electrical and Energy, Kayseri University, Kayseri, Turkey

2 WCGEC, University of California Riverside, Riverside, CA, USA

3 Faculty of Electrical Engineering, Istanbul Technical University, Istanbul, Turkey

4 Faculty of Engineering and Architecture, Department of Electrical and Electronics Engineering, Batman University, Batman, Turkey excess of deformed products affects the quality negatively. In order to increase the product quality and production capacity, it is necessary to plan the production process by considering the causes of deformations and to carry out elimination studies. For this, it is a necessity that the detection of products with invisible deformations can be done quickly [1-3].

Faults and cracks in ceramic materials may have various reasons. These deformations can be determined by examining the structural properties of ceramic materials. In the ceramic industry, test systems are rarely used to detect the presence of defects in ceramic tiles. The defects in a ceramic body $[4,5]$ are usually caused by faulty process parameters or uncontrolled raw materials during the pressing process [6]. Such defects generally include delaminating heterogeneous materials or agglomerates which reduce structural strength. Different methods are used in the detection of cracks in ceramic, porcelain or cemented materials. Kesharaju and Nagarajah used the PSP (Particle Swarm Optimization) method for fault detection in armor ceramics [7]. Morscher and Han examined the differences in the acoustic emission waveform to predict 
the failure development for composite materials tested under tensile stress fatigue conditions at different frequencies. They concluded that failure development can be estimated by differences in the acoustic emission (AE) waveform [8]. Whitlow et al. predicted the final failure location in ceramic composites using analysis of data obtained with the combination of $\mathrm{AE}$ and digital image correlation (DIC) [9]. Existing expensive inspection systems (such as X-ray methods), which can detect information about the internal structures of ceramic materials, determine the experimental selection of optimal production parameters in most cases. In many technological sectors, the infrared (IR) examination is used for the convenience, full capacity, internal application and the continuously declining costs of thermographic systems, for damage detection and analysis. Thermographic techniques [10] are generally applied to building inspections, process controls and monitoring, predictive maintenance, assessment of material properties and medical diagnosis [11].

Deep machine learning methods have emerged as a new and effective alternative to the solution of classification and estimation problems in recent years. The DCNN method, one of the most effective methods of deep machine learning, has recently been used successfully in the diagnosis of diseases in medicine, renewable energy sources, biomedical image and pattern recognition and classification problems [12-16]. Dung and Anh [17] used deep convolutional neural network models for the detection of cracks in reinforced concrete structures and reached $90 \%$ accuracy. Chetouani et al. [18], performed the classification of different types of ceramic particles using a convolutional neural network with an accuracy of $83 \%$. Konda et al. [19], determined the microstructure properties of ceramics by using convolutional neural networks and estimated ionic conductivity in their study.

The convolutional neural network (CNN) method is mentioned in the state of the art as one-dimensional CNN (1D-CNN) or two-dimensional CNN (2D-CNN), depending on the size of the data set presented to the input layer. The numbers that make up a graph (such as a noise graph) are the data used for training and testing the 1D-CNN model. In 1D-CNN, the operations take the form of a sequence, and 1D-convolution sequences are only linear weighted sums of 1D-data sequences. Any CPU (Central Processing Unit) implementation in a standard computer is sufficient and relatively fast to train compact $1 \mathrm{D}-\mathrm{CNNs}$ containing several hidden layers (e.g., 3 or less) and neurons (e.g., $<30$ ). On account of their low computing requirements, compact $1 \mathrm{D}-\mathrm{CNNs}$ are particularly appropriate for real-time and low-cost applications in mobile or handheld devices [20]. Even though 1D-CNN models exterminate the requirement of manual feature extraction, they can only generate a trustworthy prediction only with a larger number of data, because just like artificial neural networks, input data consists of one-dimensional numerical data.

The application of $2 \mathrm{D}-\mathrm{CNN}$ is different compared to $1 \mathrm{D}-\mathrm{CNN}$ as it needs more complex computation. The 2DCNN model is also known as deep CNN (DCNN) in the state of the art. Since DCNN neurons are sparsely connected with connected weights, DCNNs can operate major inputs with large computational efficiency compared to traditional fully connected MLP networks. DCNNs are irresponsive to small transmutations in input data such as translation, scaling, irregularity and twist, and can adapt to dissimilar input sizes.

Each hidden neuron in a traditional MLP or a 1D-CNN contains scalar weights, input and output. However, because of the two-dimensional nature of the images, every neuron in a DCNN includes 2D planes for weights known as kernels and input and output known as a feature map. Although DCNNs have performed very well in many fields where visual data can be used, compact 1D-CNNs with low calculation necessities have been preferred over DCNNs in recent years. The main reason for this is usually the requirement for private hardware setup [e.g., Cloud computing or Graphics Processing Unit (GPU)] to train DCNNs. Other significant reasons may be that mathematical expressions and computations in DCNNs are more complicated in matrix form, and more significantly, graphics such as noise signals are not convenient for DCNNs [20].

It is not feasible to train and test DCNN directly with 1D signal data. To do this, it is essential to transform 1D signals to $2 \mathrm{D}$ signals. Effective use of DCNNs is essential for important project studies such as $100 \%$ accuracy of cracked plates and graphic monitoring and transition to future online DCNN studies. However, choosing DCNNs may not seem advantageous in terms of both the increased workload that slows down the computer and the possible long prediction process. It is preferable to make feature extraction prior to classification rather than directly training the classifier model with raw signal data samples.

It is known that DCNN performs better than 1D CNN even for one-dimensional data. However, in random weight initiated 1D CNN, the accuracy of 1D CNN with 1D signal input may be higher than a DCNN with 2D image input. Since the number of input parameters in 1D-CNNs is much less than DCNNs, the DCNN model with more parameters and higher complications is more likely to encounter overfitting trouble than $1 \mathrm{D}-\mathrm{CNN}$. The overfitting trouble all the time negatively influences the accuracy and stability of DCNN models and this issue requires to be eliminated [21]. For this reason, in this study, we are applying an AlexNet-like network model, initializing with weights trained on imageNet, to lighten this overfitting trouble 
encountered in DCNNs. In this way, we further increase the accuracy and robustness of the DCNN model applied for the classification by making the 1D signals (noise signals) compatible with DCNN and fine-tuning the model with the transfer learning techniques. In this study, we use the short-term recorded graphical images to convert onedimensional noise signal data (1-D) into two-dimensional data (2D). To test the robustness of the pre-trained Alexnet DCNN model, classification was performed in ten classes (c1 to c9 and s1) and a tenfold cross-validation technique was used. For this, a pre-trained DCNN Alexnet model was used by using the transfer learning method. To our knowledge, this is the first successful study where the DCNN pre-trained Alexnet model has been trained and tested with visual noise data using a transfer learning technique.

Quality filtering of image data can provide high performance in studies that can be performed with DCNN models when filter design problems are overcome. Therefore, it should be considered that scientific studies on filter design problems contribute to the current success of DCNN models. Zhang et al. [22] examined the finite-time asynchronous consuming filter design problem for conical nonlinear Markov jump systems. Another important issue for deep learning algorithms is learning control, which contributes to the development and performance of learning algorithms. Iterative learning control, which is used continuously especially in deep learning methods, can positively change the performance of the learning process. Tao et al. [23] developed an iterative learning control algorithm that is more efficient considering different initial conditions. In another study by Tao et al., they developed an iterative learning control algorithm for discrete systems with multiple time delays exposed to polytopic uncertainty and limited frequency domain [24]. In another study for the development of the learning algorithm, Xin et al. [25] designed an online modeless integral reinforcement learning algorithm to solve multiplayer nonzero-sum games.

After examining the studies in the literature, it is understood that there is a need for further study and progress in the automatic crack detection of ceramic materials. In this study, a pre-trained DCNN model is used for automatic crack detection in ceramic plates. Impact noise was produced first for cracks in ceramic plates. Impact noise was then converted into numerical data and transferred to pc to achieve noise curves. A pendulum was used to obtain impact noise. The pendulums, in accordance with the dynamic system, have the same effect as the same movements [26-28]. The rest of the article is organized as follows: in the second section, the method used in the study, obtaining and arranging the data set and the architecture of the DCNN model are explained. In the third section, the results obtained from the study are presented.
In the fourth section, the results are discussed. In the fifth section, the results of the study, future studies and recommendations are presented.

\section{Methodology}

\subsection{Obtaining the data set}

In this study, an impact pendulum was used to produce a stable impact. Impact pendulum is an advanced pendulum model used to create equal effect magnitude $[28,29]$. The equation of motion of a pendulum can be obtained using Eqs. (1), (2) and (3) using Fig. 1. Consider that the damping and thrust forces are parallel to the movement of the pendulum. Let the driving force be a function of time. $D=b v$, the damping force therefore depends on the velocity $v$ or $\mathrm{r} \dot{\theta}$.

$\Gamma=r \times F$

- damping $_{\text {force }}=$ gravity $_{\text {force }}+$ driving $_{\text {force }}=I \ddot{\theta}$

$-b v r \sin \theta+-m g r \sin \theta+F r \sin \theta=I \ddot{\theta}$

By rearranging and modifying equality (4) and (5) are obtained. Equation (5) is a quadratic differential equation that identifies the dynamic system of interest [26-28].

$m r^{2} \theta+\ddot{b r^{2}} \dot{\theta}+m r g \sin \theta=F(t) r$

$\ddot{\theta}+\frac{b}{m} \dot{\theta}+\frac{g}{r} \sin \theta=\frac{F(t)}{m r}$

By means of a small plastic hammer attached to the end of the impact pendulum, an equally large impact was applied without damaging the ceramic plate and the sound from the plate was analyzed. Measurement and data collection are shown in Fig. 2.

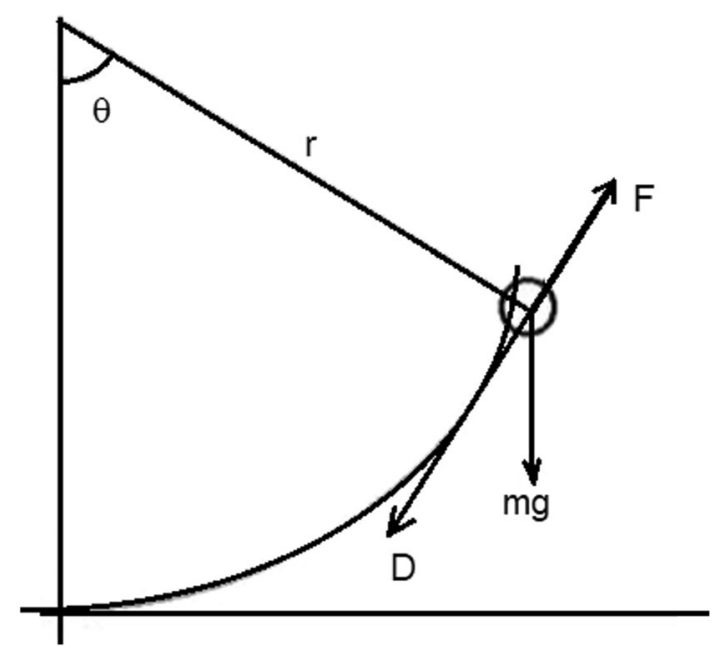

Fig. 1 Scheme of a pendulum [24] 
Fig. 2 Data acquisition and measurement systems $[28,29]$

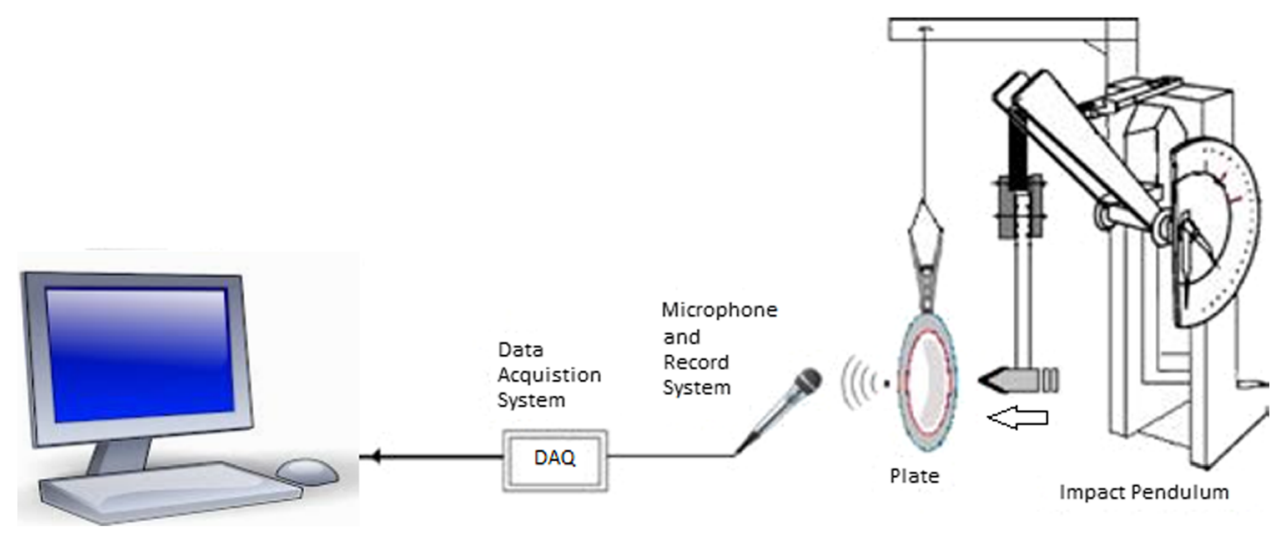

In the study, POE 2000 type Impact Pendulum was used and the same size impact effect was obtained on the ceramic plate. Here, using the impact pendulum, the sound produced as a result of equal impact on the same model cracked or non-cracked plates was transferred to the data acquisition system and then to the computer. Onyx 800R sound system model was used for experimental application. The output audio data of the amplifier were transmitted to the computer via the Advantech 1716L multifunction PCI card at a sampling rate of $0.0000125 \mathrm{~s}$. Data analysis was performed using MATLAB (Fig. 2).

A total of four ceramic plates were identified, one of which was in good condition and the others with different cracks, and in experimental application, equal impacts were applied to these plates. According to the obtained sound graphs, there is a sound absorption between the crack openings in the crack plate after the impact operation and the vibration does not continue. On the contrary, on the undamaged plate, vibration continues for a longer period of time. This shows a similar trend in other graphs, but varies according to the size and shape of the cracks in the plates. The plate used in each experiment has the same structural characteristics and is the same brand product. However, each cracked plate has invisible cracks which are different in size. Undamaged plates were called "s" and cracked plates were called "c".

The curves of the sound from the ceramic plates at the same impact are shown in Figs. 3. The last graph in Fig. 3 belongs to the undamaged plate illustrated as s1. From these graphs, a data set consisting of 2000 images was created, which will be used for the training and testing of the DCNN model by creating short-term instant graphic images. The basic numbers related to this data set are shown in Table 1.

Two hundred of the visual data were obtained from the undamaged plate with the code name s1, and the remaining 1800 visual data were obtained from cracked plates with the code names c1, c2, c3, c4, c5, c6, c7, c8 and c9. Since there are nine types of cracks, ten group datasets were created. For each group of this data set, $80 \%$ was used for the training of the model and the rest was used for validation or evaluation purposes. All the images in the data set are $547 \times 1110 \times 3$ in the first stage. These visual data were cropped to obtain dimensions of $227 \times 227 \times 3$ calculated for the pre-trained Alexnet DCNN model.

\subsection{Architecture of the DCNN model}

In this study, we used the pre-trained DCNN AlexNet model for fault detection in ceramic plates. For this architecture, MATLAB has been preferred, providing an efficient environment for deep learning. We used the concept of transfer learning for deep neural network architecture. Transfer learning is a machine learning technique in which a model trained for a particular task can be used to learn the new task by transferring information. When we do not have enough data to train the model from scratch, this technique can be effective, fast and convincing. Input images for AlexNet were Red Green Blue (RGB) color images with a resolution of $227 \times 227$ pixels. The Alexnet model consists of three maxpolling layers and five convolution layers. Each convolution layer in AlexNet architecture is followed by a rectified linear unit (ReLU). All parameters including filter size, number of filters, padding and stride for each layer are shown in Fig. 4. After removing these layers, we reorganized this architecture for crack detection in ceramic plates. To this end, instead of the fully connected layer with 1000 neurons removed, another fully connected layer with ten neurons was added by using the softmax function, to which all units were fully connected to the ten neuronal outputs. The architecture applied for the detection or classification of cracks in ceramic plates is shown in Fig. 4. The network change rate is usually carried out with a learning rate. In this network, we have not changed the learning rate of the layers before the last three layers; instead, we have increased the learning rate of the new layers to update faster than the original layers, so we can learn the new weight of these layers 

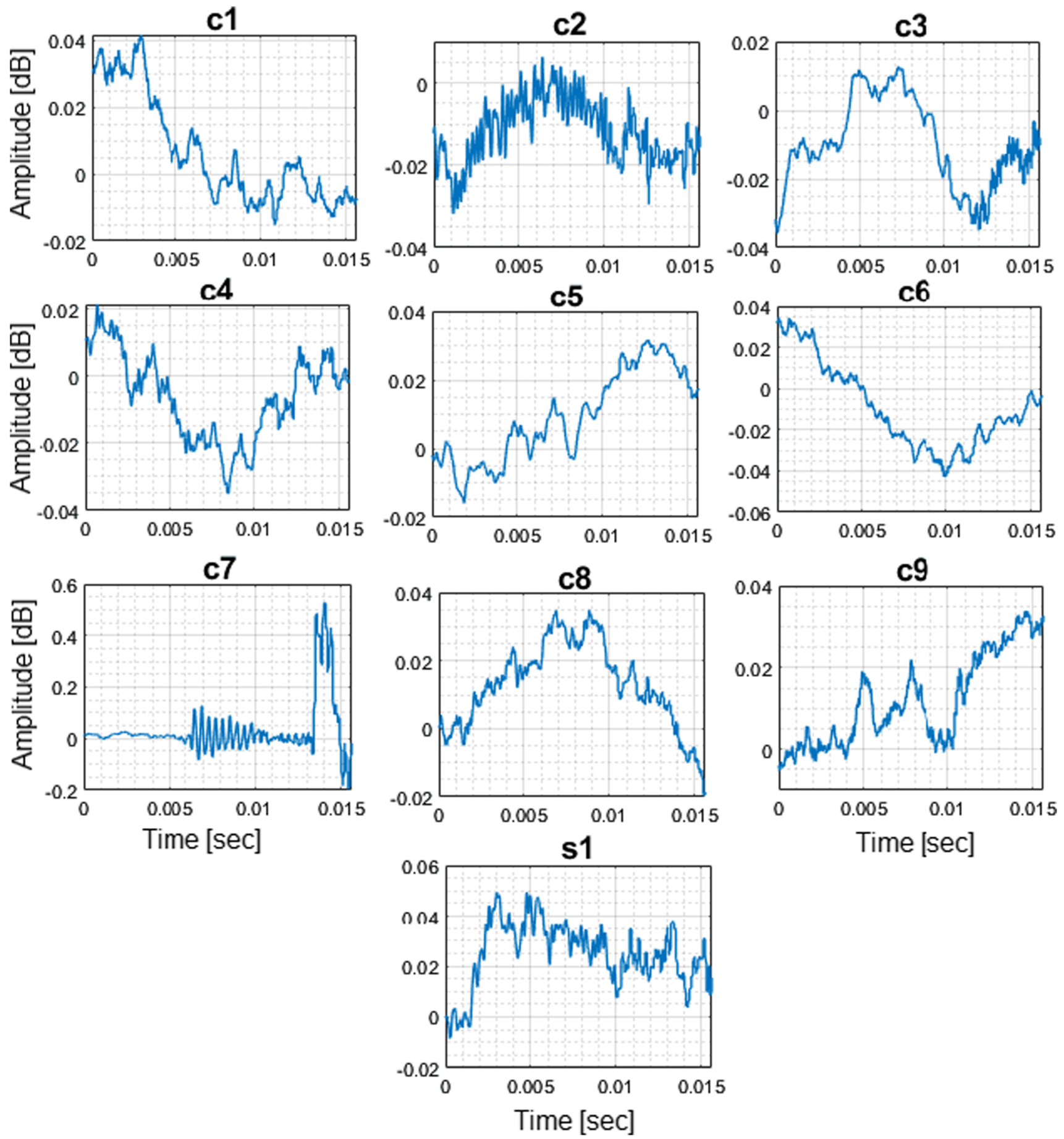

Fig. 3 Noise plot of the plates

faster. Figure 4 illustrates the pre-trained Alexnet DCNN model and the changes made. The study was carried out with the NVIDIA GeForce 940MX, 6040 MB GPU notebook. The training took about $23 \mathrm{~min}$. Batch size 75 , maximum epoch 30 and maximum iteration set to 250 . The initial learning rate was chosen as 0.001 . In this study, the pre-trained Alexnet deep convolutional neural network model was reorganized with a transfer learning approach.

\subsection{Convolution layer}

The process of filtering the input matrix with the filter matrix $\left(n_{k} \times n_{k}\right)$ is called convolution. During the 
Table 1 Data set summary

\begin{tabular}{lllll}
\hline Plates names & Situation & Data number & Training data & Validation data \\
\hline c1 & Cracked & 200 & 160 & 40 \\
c2 & Cracked & 200 & 160 & 40 \\
c3 & Cracked & 200 & 160 & 40 \\
c4 & Cracked & 200 & 160 & 40 \\
c5 & Cracked & 200 & 160 & 40 \\
c6 & Cracked & 200 & 160 & 40 \\
c7 & Cracked & 200 & 160 & 40 \\
c8 & Cracked & 200 & 160 & 40 \\
c9 & Cracked & 200 & 160 & 40 \\
s1 & Undamaged & 200 & 160 & 40 \\
\hline
\end{tabular}
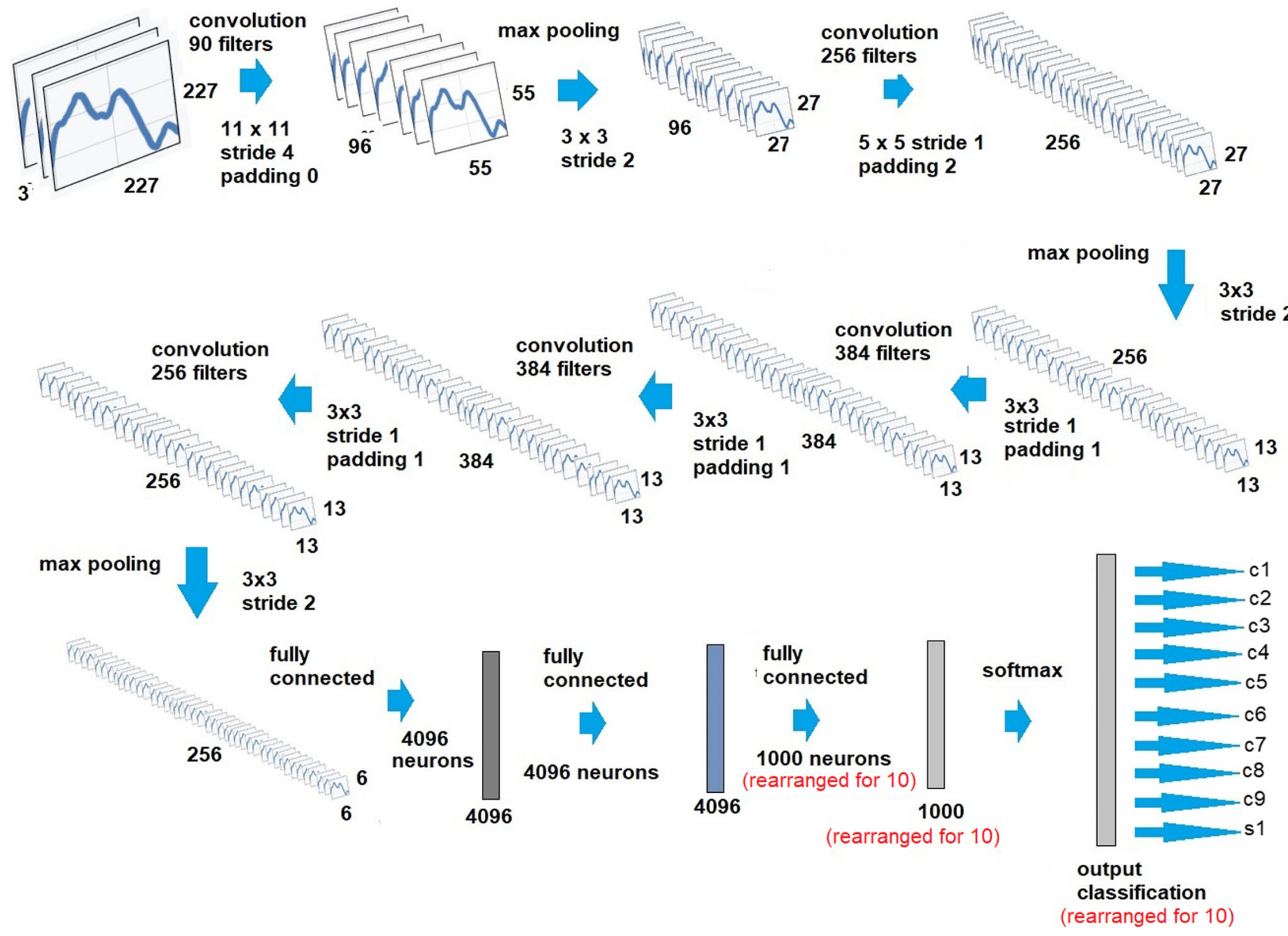

Fig. 4 AlexNet architecture for defect detection in ceramic plates

convolution process, the number of steps the filter matrix makes to the right and left in the input matrix (tensor) is called the stride (s). The input matrix $A$ is a square matrix and the row and column size is greater than or equal to $n_{k}$. The matrix $A$, which is the input matrix to the convolution layer, with dimensions $n_{A} \times n_{A}$, is converted into a smaller matrix $B$, after filtering with the filter matrix. According to Eq. (6), $K$ indicates the filter, $A$ indicates the input matrix and $B$ indicates the filtered output matrix. The output matrix $B$ obtained after filtering is obtained in the following way $[30,31]$.

$B_{i j}=(A * K)_{i j}=\sum_{f=0}^{n_{K}-1} \sum_{h=0}^{n_{K}-1} A_{i+f, j+h} K_{i+f, j+h}$ 
After convolution, the image size becomes smaller than the original size. In another convolution process, the image size can be increased to its original size if deemed necessary. For this, it is necessary to increase the size of the reduced image in pixels. This size increase process is called padding $(p)$. The blank pixel locations of the enlarged image for padding are filled with near zero pixel values or zero values. It can be applied in various different strategies for padding process. One of the steps of designing a DCNN architecture is to determine $p$ values. Another design step is to determine the $s$ value, which is the shifting step of the filter. After the $p$ and $s$ values are determined, the output matrix $B$ can be calculated as follows after the convolution and pooling processes [30, 31].

$n_{B}=\left\lfloor\frac{n_{A}+2 p-n_{K}}{s}+1\right\rfloor$

\subsection{Classification and softmax layer}

The cross-entropy loss is calculated in the classification layer, the last layer of the network, connected to the softmax layer. For the probability distribution of multi-class classification; $0 \leq y_{r} \leq 1$ and $\sum_{j=1}^{k} y_{j}=1 . a_{r}$, indicates the conditional probability of the sample in class $r$. Softmax function, which is the layer connected to the last fully connected layer, is the output unit activation function used for multiple classifications. In this study, the softmax function used for decimal classification:

$y_{r}(x)=\frac{\exp \left(a_{r}(x)\right)}{\sum_{j=1}^{k} \exp \left(a_{j}(x)\right)}$

In the training process of the model, the input from the softmax function in the classification layer is assigned to one of the $K$ classes that are mutually exclusive by means of the "cross-entropy function". The model continues to be trained until the loss function reaches its minimum value. In the loss function, $N$ indicates the sample number, $K$ is the class number, $t_{i j}$ is the output for $j$ class $i$ sample, and $y_{i j}$ is the output for $j$ class $i$ sample [31,32].

Loss $=-\sum_{i=1}^{N} \sum_{j=1}^{K} t_{i j} \ln y_{i j}$

\section{Results}

The pre-trained DCNN Alexnet model was used for crack detection in ceramic plates. We analyzed the results obtained by training the proposed pre-trained Alexnet DCNN model with the data set. Table 2 shows the results
Table 2 Classification results

\begin{tabular}{lll}
\hline & Accuracy $(\%)$ & Loss \\
\hline Training & 100 & 0.0002 \\
Validation & 99.50 & 0.0144 \\
\hline
\end{tabular}

obtained from the DCNN model. The pre-trained DCNN model has provided satisfactory accuracy for crack detection in ceramic plates. The validation accuracy rate was $99.50 \%$.

Figure 5 shows the process of accuracy rate for training and validation obtained according to iteration. Also, the loss curves in the training and validation process are illustrated in Fig. 5. The success of the DCNN model can be understood from the accuracy graphs showing the training processes, and the confusion matrix in Fig. 6.

As can be seen from the confusion matrix, only two images are incorrectly estimated. In fact, this image was s1 but it was estimated as c2. All other image data were accurately predicted, resulting in a satisfactory success in the cracked plate and undamaged plate detection.

\section{Discussion}

Using DCNN, we have performed the automatic detection of invisible cracks in the ceramic plates and examined the plate condition in ten classes. The proposed method has been applied effectively. It is understood that the DCNN model is more cost-effective compared to infrared (IR), thermographics, $\mathrm{X}$-ray methods for examining the internal structures of ceramic materials, it gives more rapid results and is more likely to be preferred in ceramic technology in the future. Because other methods help in the optimal selection of the production parameters of ceramic materials in most cases. The convolution and other hidden layers in the DCNN model used in the study are strong enough to automatically detect and classify the graphical image data set. Deep neural networks often required large amounts of data for training. However, in this study, we were able to obtain $99.50 \%$ accuracy in cracked plate detection by using short-term graphical image data and fine-tuning of pretrained DCNN despite our limited data set. The absence of a significant difference between the training accuracy and validation accuracy indicates that the DCNN is strong enough to detect cracks that cannot be seen in ceramic materials based on graphics from noise data. A relatively small amount of work in the art suggests detection techniques for different ceramic cracks, but most studies have also neglected the classification of crack subtypes. 
Fig. 5 Accuracy and loss curves for training and validation

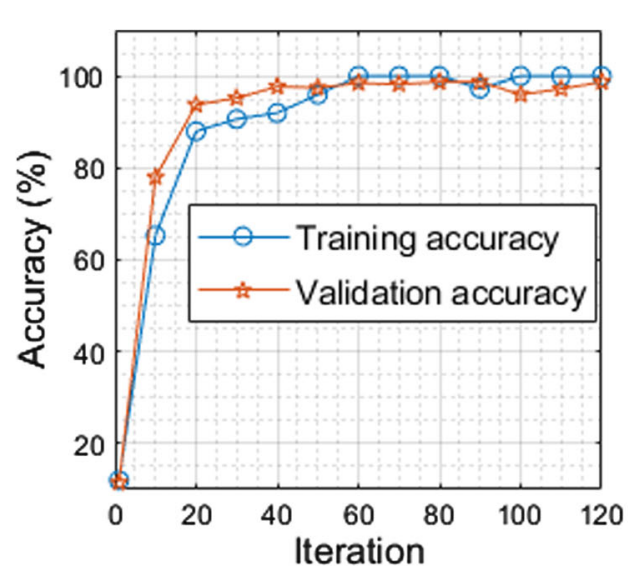

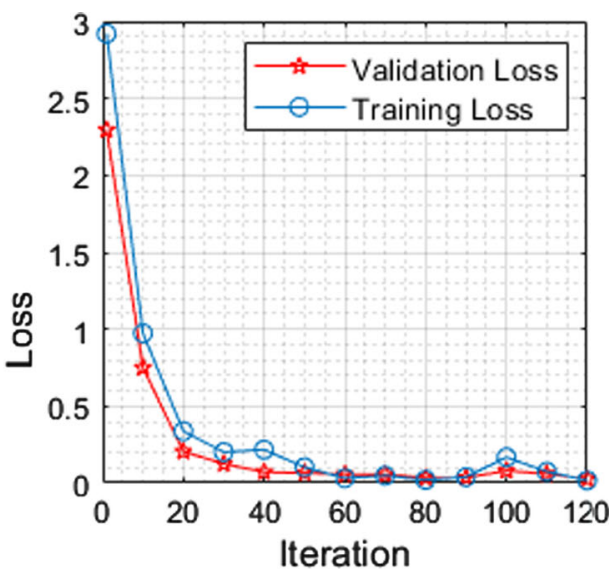

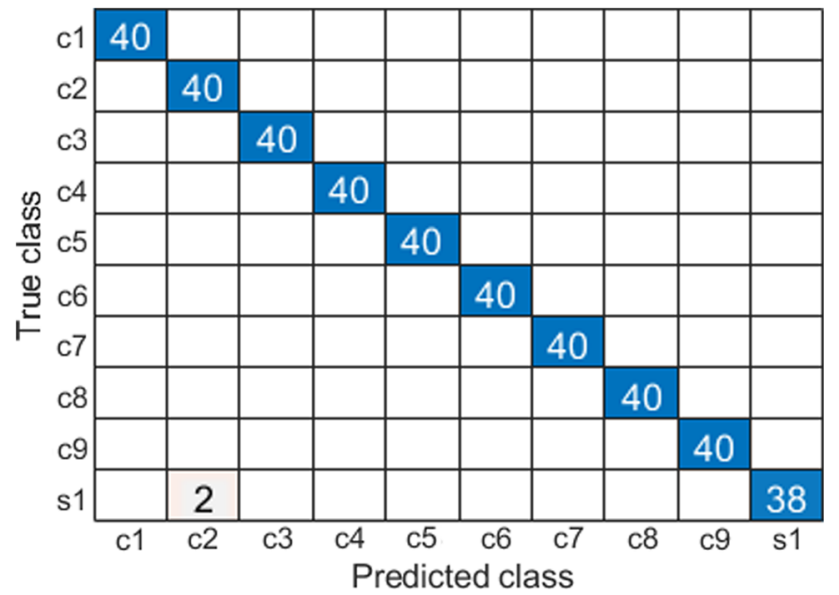

Fig. 6 Confusion matrix

Although these subtypes are difficult to use, they are of great importance in deformation detection or prediction.

In this preliminary study, automatic detection of cracked and undamaged ceramic plates by the effect of noise and classification of them into ten classes based on instant graphic images were performed. The system we have investigated in this study is the model of DCNN which is based on the instantaneous waveform image data without requiring any processing of noise data, such as feature extraction from the graph. The classification rate achieved with the DCNN pre-trained model provides the highest accuracy rate among all the methods discussed in the literature.

In our previous study, using numerical data, two undamaged and two cracked ceramic plates were predicted with an accuracy rate of approximately $99 \%$ by way of quad classifications. However, the number of crack plates we used in this study was only two, and we used only numerical vibration data of these two crack plates [33]. Therefore, the previous study needed improvement in terms of both reliability and generalizability. This study, on the other hand, was different from the previous one, using nine crack plates instead of two, and the number of crack types was increased by making ten classifications instead of four classifications. Thus, with two-dimensional visual data and deep learning, both the reliability of the study was increased and the generalizability was improved by increasing the number of crack types. Thus, we carried our previous work to a further point.

One of the limitations of our study is that we skip noise from images, but this has little effect on the performance of our proposed system. However, there are different ways to reduce noise from images that can improve the performance of the model. Some of these methods are mean, unsharp filters, Gaussian smoothing, conservative smoothing and frequency filters. In addition, in this study, we used a limited amount of training and validation data that could affect the training process of deep neural networks. Therefore, in the future, we plan to use DCNN to learn from scratch with larger image data sets to help researchers and employees effectively detect defects in ceramic or porcelain materials so that this identification system can be used in the industry.

Another limitation of our study is that the classification of cracked plates according to their causes of cracked formation was neglected in our study. Because the cause of the deformation on the plate may be due to production, raw material selection or human-induced. In real applications, we can use deep learning to reveal the cause of the crack. Thus, we can directly contribute to production.

\section{Conclusion and future direction}

In this study, we investigated the pre-trained DCNN AlexNet application for the detection of cracks in the ceramic plates, the detection and classification of the subtypes of different cracks. By performing data set creation from short-term graphical visual images, we are able to achieve $99.50 \%$ accuracy in the validation process, and 
$100 \%$ accuracy in the training process, for crack detection. This automatic identification and classification system can help to identify the early deformation of ceramic materials so that an opportunity for effective early prevention is achieved. In the future, one of the promising aspects for researchers is to use different deep learning architectures to classify and identify a variety of failures in ceramic materials and to compare these architectures to check which network performs well for deformation detection. In addition, we can apply deep learning models to learn from scratch with larger image data sets, so that we can help researchers and industry workers better identify deformations in ceramic materials through this detection system. This approach can also be developed into a fully automatic deformation detection system by defining the input-output parameters and integrating it with a fully automatic deformation detection system as part of the sub-module. Another aspect for researchers and the future is to develop an automatic detection system for the phenomenon of cracks and deformation so that all types of deformation can be automated.

\section{Declarations}

Conflict of interest The authors declared that they have no conflicts of interest in this study. We declare that we do not have any commercial or associative interest that represents a conflict of interest in connection with the work submitted.

Open Access This article is licensed under a Creative Commons Attribution 4.0 International License, which permits use, sharing, adaptation, distribution and reproduction in any medium or format, as long as you give appropriate credit to the original author(s) and the source, provide a link to the Creative Commons licence, and indicate if changes were made. The images or other third party material in this article are included in the article's Creative Commons licence, unless indicated otherwise in a credit line to the material. If material is not included in the article's Creative Commons licence and your intended use is not permitted by statutory regulation or exceeds the permitted use, you will need to obtain permission directly from the copyright holder. To view a copy of this licence, visit http://creativecommons. org/licenses/by/4.0/.

\section{References}

1. Krimsky E et al (2019) Quantification of damage and its effects on the compressive strength of an advanced ceramic. Eng Fract Mech 208:107-118

2. Munz D, Fett T (1999) Ceramics. Mechanical properties, failure behaviour, materials selection. Springer, Berlin

3. Davidge RW (1979) Mechanical behaviour of ceramics. Cambridge University Press, London

4. De Andrade RM, Paone N, Revel GM (1998) Non destructive thermal detection of delamination in ceramic tile. In: Proceedings of the ENCIT 98, Rio de Janeiro, pp 727-731
5. De Andrade RM, Esposito E, Paone N, Revel GM (1999) Nondestructive techniques for detection of delamination in ceramic tile: a laboratory comparison between IR thermal cameras and laser doppler vibrometers. Proc SPIE 3585:367-377

6. Revel GM, Rocchi S (2006) Defect detection in ceramic materials by quantitative infrared thermography. In: 8 th conference on quantitative infrared thermography-QIRT'2006, Padova, Italy.

7. Kesharaju M, Nagarajah R (2017) Particle Swarm Optimization approach to defect detection in armour ceramics. Ultrasonics $75: 124-131$

8. Morscher GN, Han Z (2018) Damage determination in ceramic composites subject to tensile fatigue using acoustic emission. Materials 11(2477):2-18

9. Whitlow T, Jones E, Przybyla C (2016) Failure Prediction in Ceramic Composites Using Acoustic Emission and Digital Image Correlation. In: 42nd annual review of progress in quantitative nondestructive evaluation AIP conference proceedings

10. Maldague XPV (2001) Theory and practice of infrared technology for nondestructive testing. Wiley, New York, pp 238-250

11. Ranachowski P, Rejmund F (2008) Mechanical acoustic examination of ceramic material. In: Proceedings of the 7th international conference EEEIC 08, Cottbus, pp 11-13

12. Shafique S, Tehsin S (2018) Acute lymphoblastic leukemia detection and classification of its subtypes using pretrained deep convolutional neural networks. Technol Cancer Res Treat 17:1-7

13. Ju Y, Sun G, Chen Q (2019) A model combining convolutional neural network and LightGBM algorithm for ultra-short-term wind power forecasting. IEEE Access 7:28309

14. Zang H, Cheng L, Ding T et al (2018) Hybrid method for shortterm photovoltaic power forecasting based on deep convolutional neural network. IET Gener Transm Distrib 12(20):4557-4567

15. Park E et al (2018) Application of the deep convolutional neural network to the forecast of solar flare occurrence using full-disk solar magnetograms. Astrophys J 869:91

16. Pang $S$ et al (2019) A novel fused convolutional neural network for biomedical image classification. Med Biol Eng Comput 57:107-121

17. Dung CV, Anh LD (2019) Autonomous concrete crack detection using deep fully convolutional neural network. Autom Constr 99:52-58

18. Chetouani A, Debroutelle T et al (2018) Classification of ceramic shards based on convolutional neural network. In: 2018 25th IEEE international conference on image processing (ICIP)

19. Kondo R et al (2017) Microstructure recognition using convolutional neural networks for prediction of ionic conductivity in ceramics. Acta Metarialia 141:29-38

20. Kiranyaz S, Avci O, Abdeljaber O, Ince Gabbouj M, Inman DJ (2019) 1D convolutional neural networks and applications: a survey. https://arxiv.org/abs/1905.03554

21. Zhao J, Mao X, Chen L (2019) Speech emotion recognition using deep 1D \& 2D CNN LSTM networks. Biomed Signal Process Control 47:312-323

22. Zhang X, He S, Stojanovic V et al (2021) Finite-time asynchronous dissipative filtering of conic-type nonlinear Markov jump systems. Sci. China Inf. Sci. 64:152206. https://doi.org/10. 1007/s11432-020-2913-x

23. Tao H, Li J, Chen Y, Stojanovic V, Yang H (2020) Robust pointto-point iterative learning control with trial-varying initial conditions. IET Control Theory Appl 14(19):3344-3350

24. Tao H, Li X, Paszke W et al (2021) Robust PD-type iterative learning control for discrete systems with multiple time-delays subjected to polytopic uncertainty and restricted frequency-domain. Multidim Syst Sign Process 32:671-692. https://doi.org/10. 1007/s11045-020-00754-9

25. Xin X, Tu Y, Stojanovic V, Wang H, Shi K, He S, Pan T (2022) Online reinforcement learning multiplayer non-zero sum games 
of continuous-time Markov jump linear systems. Appl Math Comput 412:126537. https://doi.org/10.1016/j.amc.2021.126537

26. Bevivino $\mathbf{J}$ (2009) The path from the simple pendulum to chaos. Dyn Horsetooth 1:1-24

27. Kater $H$ (1818) An account of experiments for determining the length of the pendulum vibrating seconds in the latitude of London. Phil Trans R Soc (London) 104:33-109

28. Akinci TC (2011) The defect detection in ceramic materials based on time-frequency analysis by using the method of impulse noise. Arch Acoust 36(1):77-85

29. Kamilov S, Karabaeva M, Abdurrahmanov M (1998) Studies of structure of ceramic materials containing molibdenum particles within the framework of theory of non-homogeneous systems, TUBITAK. Tr J Phys 22:777-781
30. Michelucci U (2019) Advanced applied deep learning convolutional neural networks and object detection. TOELT LLC Dübendorf Switzerland First edt, pp 161-176

31. Nogay HS, Akinci CT (2020) Classification of operation cases in electric arc welding wachine by using deep convolutional neural networks. Neural Comput Appl. https://doi.org/10.1007/s00521020-05436-y

32. Bishop CM (2006) Pattern recognition and machine learning. Springer, New York NY

33. Akinci TC, Nogay HS, Yilmaz O (2012) Application of artificial neural networks for defect detection in ceramic materials. Arch Acoust 37(3):279-286

Publisher's Note Springer Nature remains neutral with regard to jurisdictional claims in published maps and institutional affiliations. 